\title{
El animal humano y su comportamiento en emergencias
}

\author{
Fernando Gordillo León y Lilia Mestas Hernández
}

\begin{abstract}
Resumen
El comportamiento de los seres humanos, como el del resto de los animales, se ve modulado por el grupo social al que pertenece. La agrupación de los seres humanos ha sido parte importante en el proceso evolutivo, al incrementar su capacidad de adaptación al medio. Si embargo, en sociedades tan amplias y complejas como las actuales, los sistemas de defensa propios del grupo se podrían volver desadaptativos. En concreto, el aprendizaje social y la rápida transmisión de la información en contextos de peligro e incertidumbre podrían generar altos niveles de activación emocional, descontrol de la situación y escasez de recursos. Una de las claves para mejorar la respuesta del grupo ante estas condiciones podría consistir en buscar el punto óptimo de activación emocional de la población, que evite tanto la subestimación como la sobrestimación de los peligros asociados con la emergencia.
\end{abstract}

Palabras clave: aprendizaje social, atajos mentales, imitación, miedo, emergencia.

\section{THE HUMAN ANIMAL AND ITS BEHAVIOR IN EMERGENCIES}

\begin{abstract}
The behavior of human beings, as other animals, is modulated by the social group to which they belong. The grouping of human beings has been an important part of the evolutionary process, increasing their ability to adapt to the environment. However, in societies as broad and complex as the ones today, the group's own defense systems could become maladaptive. Specifically, social learning and transmission of information in dangerous and uncertain contexts could generate high levels of emotional activation, lack of control of the situation and scarcity of resources. One of the keys to improve group's response could be to find the point of optimal emotional activation for the population, which avoids both underestimation and overestimation of the dangers associated with the emergency.
\end{abstract}

Keywords: social learning, heuristic, imitation, fear, emergency.

Recepción: 24/04/2020. Aprobación: 21/10/2020. Dol: http://doi.org/10.22201/cuaieed.16076079e.2021.22.1.2 
"El animal humano y su comportamiento en emergencias" Fernando Gordillo León y Lilia Mestas Hernández Vol. 22, Núm. 1, enero-febrero 2021 Revista Digital Universitaria

\section{Fernando Gordillo León}

fgordillo@ucjc.edu

http://orcid.org/0000-0001-9628-3989

Licenciado en Psicología por la Universidad Nacional de Educación a Distancia. Máster en Neurociencias por el Instituto de Neurociencias de Castilla y León. Dr. en Neurociencias (2011) y Psicología (2018) por la Universidad de Salamanca. En la actualidad es Profesor Contratado Doctor en la Universidad Camilo José Cela (Madrid, España), donde imparte las materias de Psicología de la memoria, Psicología de la emoción y la motivación, y Psicología Social. Ha participado como autor o coautor en más de 100 publicaciones tanto nacionales como internacionales, desarrollando una línea de investigación orientada al análisis de la relación entre la emoción y los procesos cognitivos.

\section{Lilia Mestas Hernández}

lilia_mestas@yahoo.com.mx

https://orcid.org/0000-0002-9819-8232

Licenciada en Psicología y Maestra en Neuropsicología por la FES Zaragoza y Dra. en Psicología en el área de Neurociencias de la Conducta por la Facultad de Psicología, unam. Experiencia en trabajo clínico y de investigación en distintos centros de atención a pacientes neurológicos y neuropsiquiátricos. Profesora de Tiempo Completo en la Licenciatura en Psicología en la Facultad de Estudios Superiores Zaragoza unam. Miembro de diferentes Comités tutoriales para la sustentación de exámenes de Posgrado y Licenciatura. Con presentación de trabajos de investigación tanto en Congresos nacionales como internacionales y cuento con publicaciones en revistas arbitradas y con factor de impacto. Colaboradora en proyectos de investigación con financiación tanto en México como en España. Miembro del Grupo de Investigación de Análisis de Comportamiento no Verbal, Madrid, España; miembro del equipo editorial de la revista Behavior and Law y revisora de distintas revistas internacionales. Editora responsable de la Revista electrónica de la FES Zaragoza en su nueva época. Miembro del Sistema Nacional de Investigadores Nivel I desde 2015 a la fecha. 


\section{Nos invaden los marcianos}

La guerra de los mundos, la novela radiofónica que impactó a los norteamericanos en el siglo pasado, supuso una de las primeras aproximaciones al estudio de las reacciones grupales. Se estima que al menos seis millones de personas oyeron la narración de Orson Welles basada en la novela de Herbert George Wells (1898), y que más de un millón creyó que se trataba de noticias verdaderas: la tierra estaba siendo invadida por marcianos (Worchel et al., 2009, video 1). El psicólogo Hadley Cantril estudió este suceso (1940) y partir de 135 entrevistas clasificó las reacciones de las personas. Encontró que entre las más comunes estaba buscar la compañía de otros; además, sugirió que el impacto de la transmisión no tuvo tanto que ver con el contenido, sino con los criterios que utilizaron las personas para evaluarlo (Tomyn, 2017). Esto nos situa directamente en la perspectiva del oyente, de la persona que valora una situación determinada.

Video 1. Transmisión original de La guerra de los mundos de 1938 [en inglés] (Orson Welles Topic, 2015).

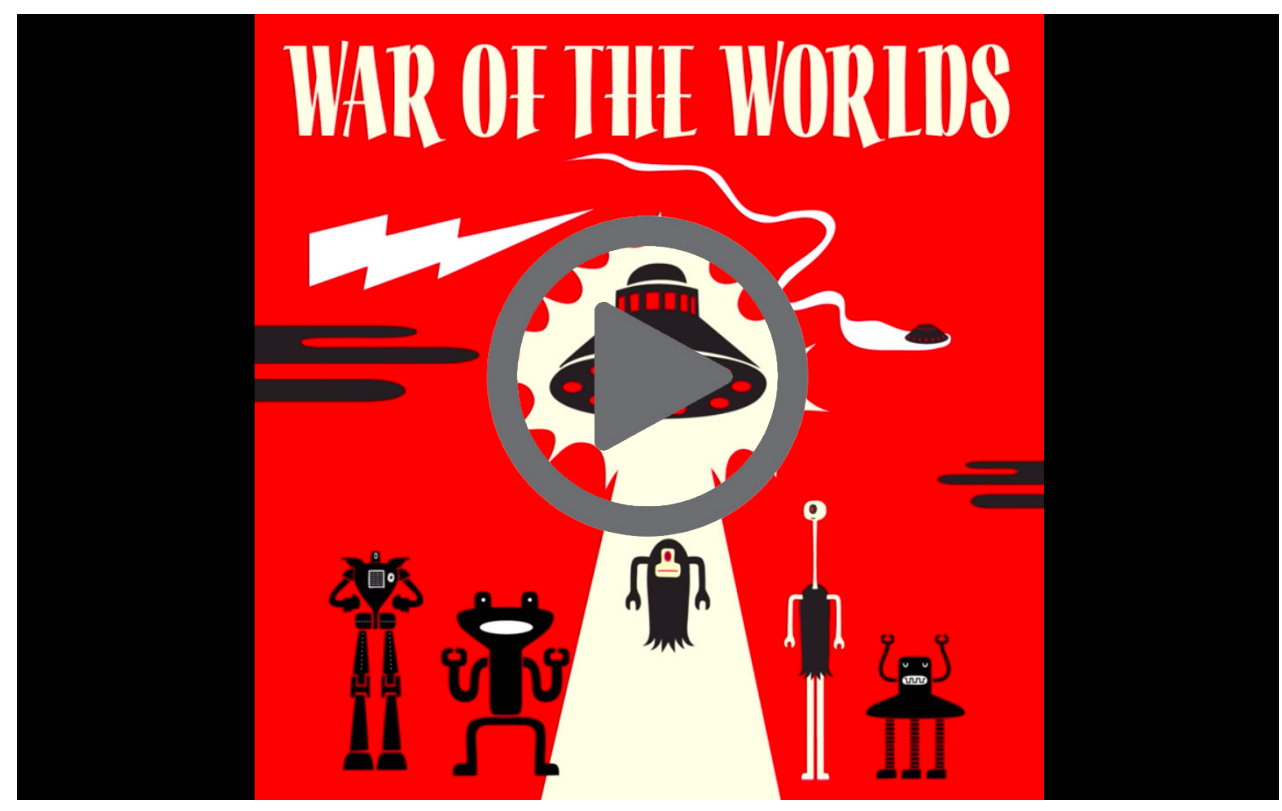

La manera en que las personas evalúan su entorno es la base explicativa de la propia emoción y, por supuesto, de trastornos psiquiátricos como la ansiedad y el estrés (Lazarus y Folkman, 1984). Estas alteraciones vendrían determinadas por dos condiciones desencadenantes, una relacionada con la valoración que el sujeto realiza de la situación ("¿es una amenaza?"), y la otra con su capacidad de afrontamiento ("¿seré capaz de superar esta situación?"). Precisamente estas condiciones se dieron en una gran parte de las personas que el 30 de octubre de 1938 oyeron desde sus casas que los marcianos estaban invadiendo la tierra, y consideraron que se trataba de una amenaza que no sabían cómo afrontar. 
Imagen . Plumerio Pipichas

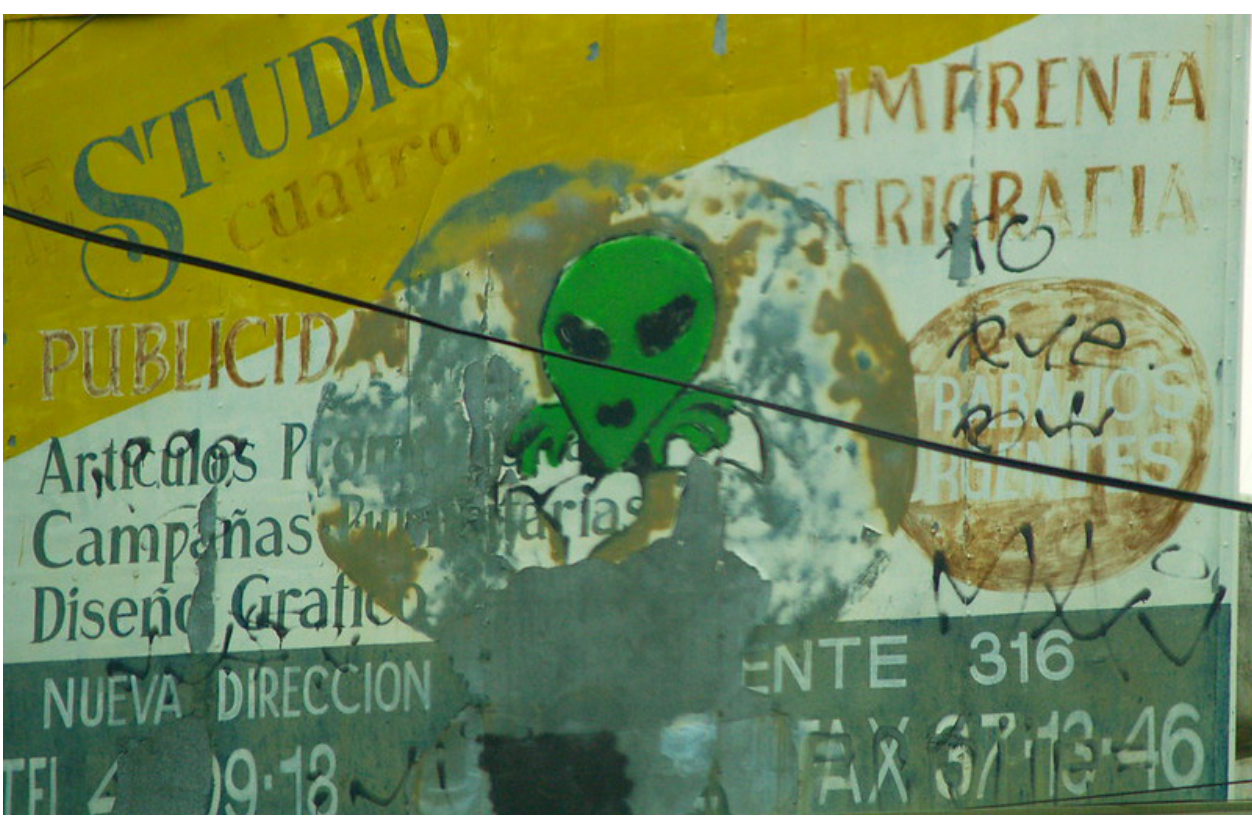

\section{El aprendizaje social: imitar para sobrevivir}

Las personas pueden comportarse de manera diferente cuando están solos a cuando pertenecen un grupo. En cierta medida, esto puede explicarse porque nuestros juicios éticos se tornan más flexibles cuando formamos parte de un grupo, lo que genera una tendencia a reconfigurar creencias y valores para adaptarlos a los del grupo (Cikara et al., 2014). De esta forma, una persona que en su vida cotidiana es respetuosa con las normas sociales podría llegar a tener conductas poco cívicas cuando se junta, por ejemplo, con los hinchas de un equipo de fútbol.

Resulta de especial interés entender cómo se comporta un grupo ante una situación de emergencia, así como las condiciones que desencadenan, mantienen e incrementan los niveles de miedo en una sociedad, una nación, o en el mundo, como está sucediendo actualmente con la pandemia causada por el SARS-CoV-2. El miedo favorece la aparición de determinados tipos de conductas, como las de imitación ("si la gente compra papel higiénico por algo será, independientemente de que tenga sentido para mí"). Este aprendizaje social, también denominado vicario, modelamiento, o aprendizaje por observación, se define como el aprendizaje de una conducta después de ver cómo un sujeto modelo la realiza (Thorndike, 1911). Una cuestión importante es la de comprender el origen de este tipo de aprendizaje, las variables que determinan su puesta en marcha, y las repercusiones que puede tener en situaciones de emergencia.

Respecto al origen de este tipo de aprendizaje, podemos obtener alguna pista al observar el comportamiento de los animales. Por ejemplo, se ha comprobado que las abejas y las palomas ante la escasez de alimentos 
incrementan las conductas de imitación y toman como sujetos modelo a sus congéneres (Lefebvre y Palameta, 1988; Grüter y Ratnieks 2011). Asimismo, las características del contexto parecen ser variables relevantes y moduladoras del aprendizaje social. Cuando la situación es peligrosa se tiende a adoptar información de tipo social (nos fijamos más en lo que hacen los demás), aunque sea menos precisa (Boyd y Richerson, 1985; Webster y Laland, 2008). De igual manera, cuando el contexto es incierto se confía más en el aprendizaje social. Así se ha observado en ratas, que realizan más conductas de imitación ante la necesidad de comer alimentos desconocidos, respecto a cuando les son familiares (Galef y Whiskin, 2004).

Por lo tanto, el aprendizaje social se incrementa, como último recurso, cuando se agotan las opciones disponibles, en contextos peligrosos e inciertos, en los que se piensa que podrían escasear los recursos (Riba, 2016). Esto permite explicar conductas como las observadas en la actual pandemia de la covid-19. Sin embargo, hay que preguntarse si en las sociedades actuales y en este tipo de situaciones la estrategia de imitar para sobrevivir es tan efectiva como en sociedades primitivas o en grupos más reducidos. Se puede pensar que no, porque existe una alta probabilidad de que la conducta que se emula no tenga suficiente base racional y, por lo tanto, su imitación masiva puede poner en peligro a todo el grupo.

Imagen . Phil Fiddyment (2015)

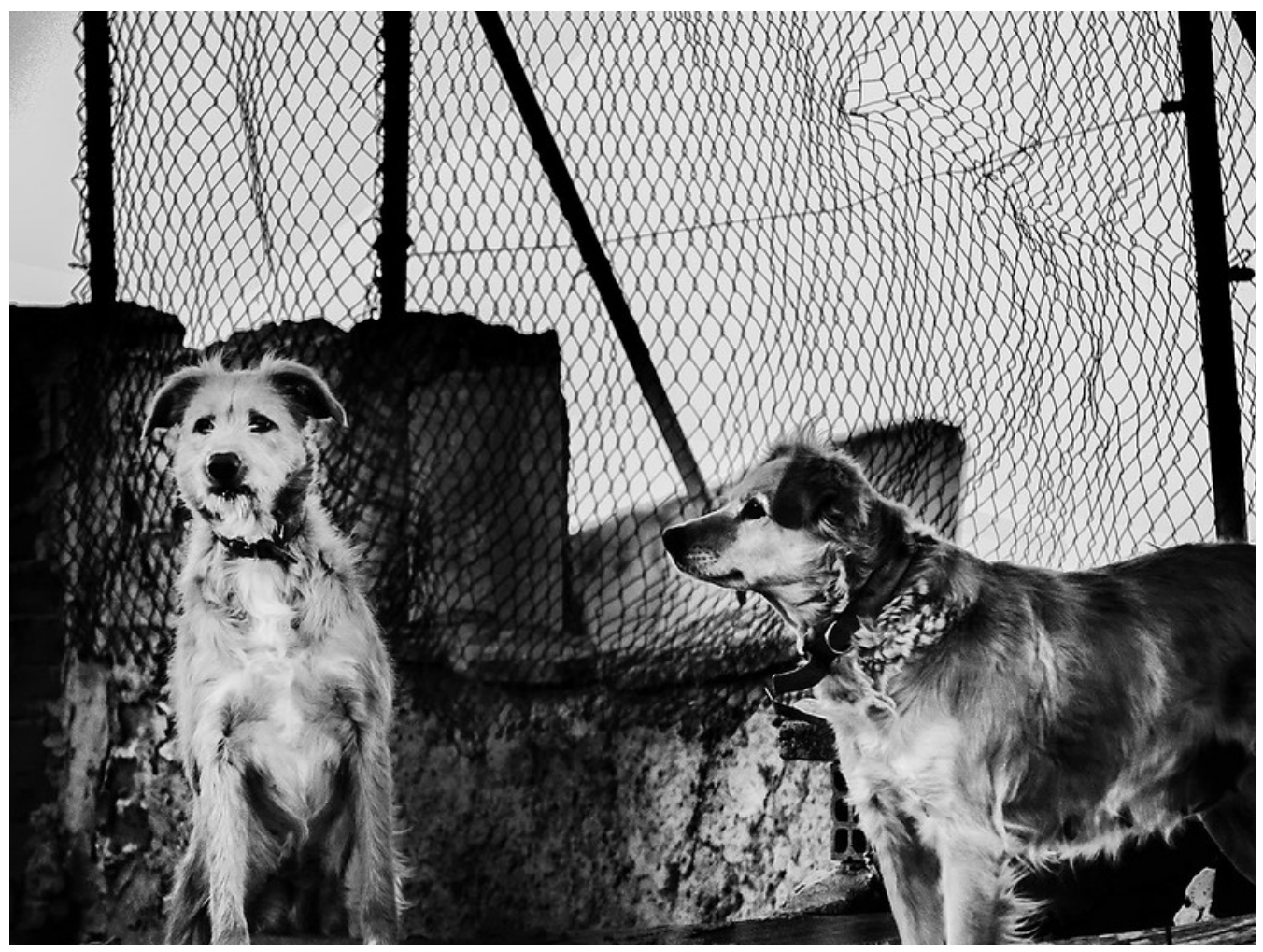


"El animal humano y su comportamiento en emergencias" Fernando Gordillo León y Lilia Mestas Hernández Vol. 22, Núm. 1, enero-febrero 2021 Revista Digital Universitaria

\section{Los medios de comunicación: el virus del miedo}

Otro de los aspectos para tener en cuenta, ya evidenciado en el episodio de $L a$ guerra de los mundos, es la gran rapidez con la que los medios de comunicación transmiten la información, lo que puede incrementar el miedo y la incertidumbre en la sociedad. En el presente, esto se ve exacerbado por los sistemas de transmisión de la información actuales, que permiten enviar un mensaje de un lado al otro del mundo en cuestión de segundos. Se ha comprobado que, en situaciones de emergencia, como terremotos o avisos de bomba, en los primeros momentos se registra una localización espacial clara de las comunicaciones, que alcanza rangos geográficos más amplios en muy poco tiempo (Bagrow et al., 2010). Por lo tanto, las noticias, verdaderas o falsas, se expanden en cuestión de horas o días a todo el mundo. Los canales de comunicación como el popular WhatsApp son un caldo de cultivo ideal para las falsas noticias (fake news), que contribuyen a incrementar el miedo y la incertidumbre en la población y pueden generar descontrol de la situación.

En la propagación de noticias falsas y alarmantes — que si bien en ciertos casos sí busca la desestabilización de la población de manera intencionadaintervienen factores que tienen que ver con la propia inercia del miedo sobre la conducta de las personas. Un receptor decide difundir una noticia posiblemente falsa y alarmante para proteger al resto, pensando que, de no ser cierta, no tendría consecuencias muy graves. Este proceso decisional se puede entender como un heurístico o atajo mental (véase Tversky y Kahneman, 1973), en tanto se decide asumir ciertos riesgos al pensar que los beneficios ante la posible certeza de la noticia serían mayores que los perjuicios ante su falsedad.

Por ejemplo, si se considera que un determinado medicamento agrava los síntomas de la enfermedad, será preferible advertir a la población aun sin saberlo con certeza, porque las muertes que se evitarían compensarían los perjuicios derivados de su falsedad. Este es el mecanismo de defensa se observa en los grupos sociales ante una situación de emergencia: se prioriza la rapidez en la respuesta (reenviar el mensaje alarmante de manera rápida), frente a la precisión (no comprobar su veracidad). Puede entenderse como un mecanismo adaptativo, en el que se prefiere cometer un error por exceso que por defecto, e incrementar así la probabilidad de supervivencia (si es cierto, habremos reaccionado rápido, si no lo es, las consecuencias no serán muy graves).

Dicho mecanismo también lo podemos encontrar a nivel de los circuitos neuronales, tal y como lo ha planteado Ledoux (1986), quien explica cómo ante la presencia de un estímulo potencialmente peligroso, la información se procesa por dos vías, una secundaria inconsciente (tálamo-amígala), y una primaria consciente (tálamo-corteza). La vía secundaria es más rápida y procesa el estímulo a partir de sus características básicas; por otro lado, en la primaria, que es más lenta, se realiza un procesamiento de la información más profundo. 
"El animal humano y su comportamiento en emergencias" Fernando Gordillo León y Lilia Mestas Hernández Vol. 22, Núm. 1, enero-febrero 2021 Revista Digital Universitaria

Por ejemplo, si fuéramos caminando por el parque y de pronto se moviera un arbusto a nuestra izquierda, de manera automática iniciaríamos la conducta de huida, porque la vía secundaria (tálamo-amígdala) habría procesado el estímulo como potencialmente peligroso, poniendo en marcha la respuesta defensiva. Sin embargo, si tras el arbusto apareciera un pequeño e inofensivo perro, la corteza cerebral, que habría procesado la información de manera más profunda, determinaría que el estímulo no es peligroso y enviaría señales a la amígdala para inhibir la conducta de huida. En ese caso, se puede observar cómo el circuito prioriza una respuesta rápida, aunque no siempre certera, que es modulada posteriormente por la información de la corteza (me asusto y empiezo a huir, pero cuando me doy cuenta de que el estímulo no es peligroso me detengo y continuo con mi paseo). Lo anterior resulta muy adaptativo, porque es preferible reaccionar rápido y equivocarse, que no reaccionar y exponerse al peligro.

Además, este sistema de defensa interactúa con otros procesos cognitivos, como la memoria (eventos pasados y memoria autobiográfica), para conformar la experiencia de miedo en contextos complejos (Ledoux, 2020). En el mismo sentido, pero a nivel de las comunicaciones sociales, se asume la misma regla: que es preferible reaccionar rápido (reenviar la noticia) y equivocarse, que no reaccionar rápido y poner en peligro la supervivencia. Así, en muchos niveles en el entendimiento de los seres humanos aplican las mismas reglas de supervivencia, sin embargo, en el ámbito social esto puede resultar claramente desadaptativo, porque no hay una vía primaria que nos permita inhibir o corregir la conducta de reenvío, y la información, ya transmitida sin control alguno, eleva en exceso los niveles de alerta de la sociedad.

Ahora bien, de lo dicho se puede inferir la necesidad de mantener el miedo de la población ante una emergencia a un nivel de activación adecuado. ¿Por qué? El miedo no es una emoción negativa sino adaptativa y, con la ansiedad, nos permite reaccionar ante la presencia de una amenaza y prever posibles peligros. No obstante, la excesiva intensidad de estas emociones puede volverlas desadaptativa. El miedo puede ser eficaz en generar cambios de comportamiento si las personas se sienten capaces de enfrentar la situación, pero también puede generar reacciones defensivas si no lo perciben de esta manera (Witte y Allen, 2000). Que se sientan capaces o no de afrontar la situación, en muchas ocasiones, dependerá de la información recibida por los gobiernos, los medios de comunicación, y por vías de transmisión de la información no contrastada, como las redes sociales.

Las consecuencias del miedo se reflejan de manera clara en un proceso básico como la toma de decisiones. En situaciones de emergencia debemos tomar muchas decisiones, y éstas se vuelve relevantes cuando sus consecuencias afectan a otras personas, por ejemplo, las que deben tomar los políticos. En este contexto, las decisiones deben ser operativas, que busquen solucionar problemas, al tiempo que estabilicen el estado anímico de la población y que procuren el punto óptimo de activación emocional, lo que facilitará el inicio y 
mantenimiento de conductas preventivas y necesarias para superar la emergencia. Si el nivel de activación emocional está por debajo del punto óptimo, gran parte de las personas no percibirá la situación como peligrosa, y si la activación es excesiva, ésta puede generar conductas irracionales e igualmente peligrosas. La búsqueda de este equilibrio no es fácil, pero sí necesaria.

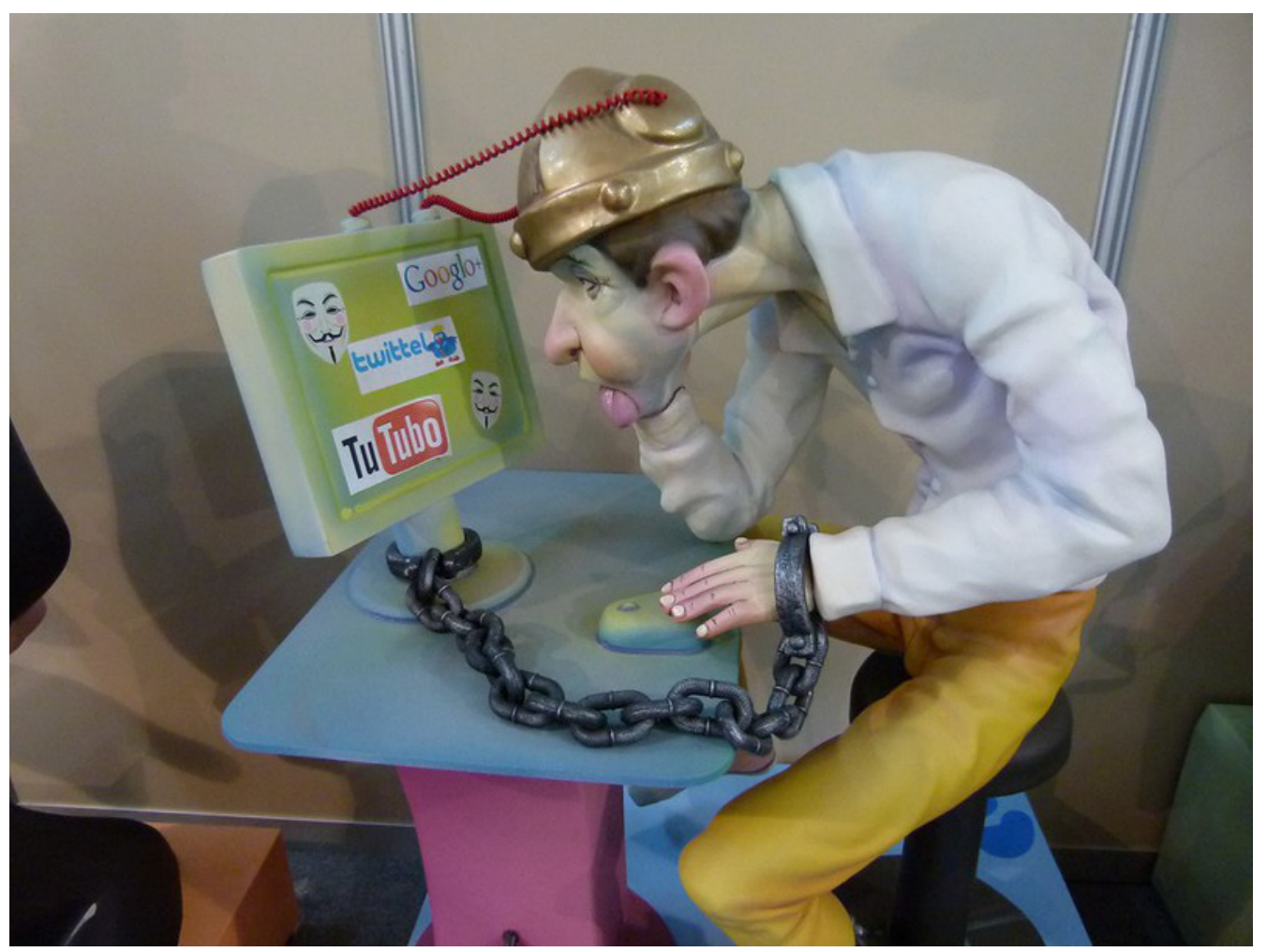

\section{Conclusiones}

En definitiva, y teniendo en cuenta todo lo dicho, para afrontar situaciones de emergencia hay que tener en cuenta los siguientes puntos:

1. Gestionar la información sobre la emergencia de manera consciente (evitar los atajos mentales en el procesamiento de la información).

2. Ser consciente de los efectos negativos del aprendizaje social sobre la conducta. Si bien se trata de una estrategia útil en determinados contextos, pues facilita una respuesta rápida ante situaciones peligrosas e inciertas, en otras circunstancias puede perjudicar la supervivencia de sociedades complejas, como la actual, con vías de transmisión de la información muy rápidas y efectivas, y donde la imitación puede generar el colapso de determinados recursos.

3. Tomar consciencia de nuestras conductas y dedicar unos minutos a razonar sobre ellas. 
"El animal humano y su comportamiento en emergencias" Fernando Gordillo León y Lilia Mestas Hernández Vol. 22, Núm. 1, enero-febrero 2021 Revista Digital Universitaria

4. Regular en lo posible los niveles de activación emocional, evitando tanto la baja activación (despreciar el posible peligro), como la excesiva activación (exagerar el peligro y sus consecuencias).

\section{Referencias}

* Bagrow, J. P., Wang, D. y Barabasi, A-L. (2010). Collective response of human populations to large scale emergencies. Plos One, 6(3), e17680. Dol: https://doi. org/10.1371/journal.pone.0017680

* Boyd, R. y Richerson, P. (1985). Culture and the evolutionary process. University of Chicago, Chicago.

Cantril, H. (1940). The invasion from Mars: a study in the psychology of panic; with the complete script of the famous Orson Welles broadcast. Princeton University Press.

* Cikara, M., Jenkins, A. C., Dufour, N. y Saxe R. (2014). Reduced self-referential neural response during intergroup competition predicts competitor harm. Neurolmage, 96, 36-43. Dol: https://doi.org/10.1016/j.neuroimage.2014.03.080

* Fiddyment, P. (2015, 3 de enero). Animals [fotografía]. Flickr. https://flic.kr/p/HFJhiS

* Galef, B. G., Jr. y Whiskin, E. E. (2004). Effects of environmental stability and demonstrator age on social learning of food preferences by young Norway rats. Animal Behaviour, 68(4), 897-902. Dol: https://doi.org/10.1016/j. anbehav.2003.10.029

* Grueter, C. y Ratnieks, F. (2011). Honeybee foragers increase the use of waggle dance information when private information becomes unrewarding. Animal behaviour, 81(5), 949-954. Dol: https://doi.org/10.1016/j.anbehav.2011.01.014

* Lazarus, R. S. y Folkman, S. (1984). Estrés y procesos cognitivo. Springer Publishing Company.

- LeDoux, J. E. (1986). Sensory systems and emotion: a model of affective processing. Integrative Psychiatry, 4, 237-248.

* LeDoux, J. E. (2020). My Word. Thoughtful feelings. Current Biology, 30(11), R617-R634. https://www.cns.nyu.edu/ledoux/pdf/thoughtful_feelings_ current\%20biology_may2020.pdf

* Lefebvre, L. y Palameta, B. (1988). Mechanisms, ecology, and population diffusion of socially learned, food-finding behavior in feral pigeons. En T. R. Zentall y B. G. Galef, Jr. (Eds.), Social learning: Psychological and biological perspectives (p. 141164). Lawrence Erlbaum Associates, Inc.

* Marín Segovia, A. (2012, 1 de marzo). Antonio Marín Segovia. Internet ha sido asesinado por el macarrismo ilustrado de Wert, con el beneplácito del PPSOE [imagen]. Flickr. https://flic.kr/p/bmM8Sj

- Orson Welles - Topic. (2015, 24 de febrero). War Of The Worlds - Original 1938 Radio Broadcasts (2011 Remastered Version) [video]. YouTube. https://youtu. be/9q7tN7MhQ4I 
"El animal humano y su comportamiento en emergencias" Fernando Gordillo León y Lilia Mestas Hernández Vol. 22, Núm. 1, enero-febrero 2021 Revista Digital Universitaria

*ipichas, P. (2007, 5 de mayo). Marciano [fotografía]. Flickr. https://flic.kr/p/Ng2iP

* Riba Cano, D. (2016). Mecanismos cognitivos de aprendizaje social en chimpancés (Pan Troglodytes): Evaluación experimental a través de múltiples tareas [tesis de doctorado, Universitat Rovira i Virgili]. http://hdl.handle.net/10803/399230

* Thorndike, E. L. (1911). Animal intelligence: Experimental Studies. MacMillan.

* Tomyn, A. J. (2017). Hadley Cantril: A Pioneer in Public Opinion Research. Applied Research Quality Life, 12, 1033-1034. Dol: https://doi.org/10.1007/s11482-0179569-9

* Tversky, A. y Kahneman D. (1973). Availability: a heuristic for judging frequency and probability. Cognitive Psychology, 5(2), 207-232. Dol: https://doi.org/10.1016/00100285(73)90033-9

- Webster, M. M. y Laland, K. N. (2008). Social learning strategies and predation risk: minnows copy only when using private information would be costly. Proceedings of the Royal Society B: Biological Sciences, 275, 2869-2876. Dol: https://doi. org/10.1098/rspb.2008.0817

* Witte, K. y Allen, M. (2000). A Meta-Analysis of Fear Appeals: Implications for Effective Public Health Campaigns. Health Education \& Behavior, 27(5), 591-615. Dol: https://doi.org/10.1177/109019810002700506

* Worchel, S., Cooper, J., Goethals, G. R., y Olson, J. M. (2009). Psicología social. Paraninfo.

\section{Cómo CITAR ESTE ARTículo}

* Gordillo León, Fernando y Mestas Hernández, Lilia. (2021, enero-febrero). El animal humano y su comportamiento en emergencias. Revista Digital Universitaria (RDU), 22(1). Dol: http://doi.org/10.22201/cuaieed.16076079e.2021.22.1.2 